\title{
Hearing loss in patients with chronic kidney disease and psychological burden in their caregivers
}

\author{
Ghana Med J 2019; 53(3): 189 doi: http://dx.doi.org/10.4314/gmj.v53i3.1
}

Chronic Kidney Disease (CKD) is assuming alarming proportion lately in Sub-Saharan Africa, a continent already saddled with infectious disease leading to the description of the continent as being hit with a double burden of diseases. ${ }^{1}$ Despite the huge challenges faced by CKD patients in this region in terms of accessibility and affordability to care, several co-morbidities exist among these patients and their caregivers also suffer numerous psychological problems which are often overlooked. The two studies published in this issue of the journal highlight the need to embrace these additional burdens amongst patients with CKD and their caregivers.

Patients with advanced CKD requiring renal replacement therapy often require assistance from other people for their activities of daily living such as housekeeping, medication administration, and transportation to and from hospital to keep up with clinic attendance and dialysis sessions. Majority of these caregivers are family members or close relations who do not get paid for their services. Some of these caregivers at times give up on their jobs to devote themselves for their ailing relations. A previous study has reported that caregivers have increased psychological problems, impaired quality of life and increased mortality. ${ }^{2}$ These caregivers have an increased risk of stress, depression, anxiety and poor quality of life in the course of providing care and support for their loved ones. ${ }^{3}$ Yet these psychological needs are often unmet because most renal teams do not fully include these caregivers in their management plan. This commentary is therefore intended to bring to the fore the need to include caregivers of CKD patients in the treatment guidelines in CKD management.

Another co-morbidity often overlooked in patients with CKD is hearing loss. Hearing loss has been found to be more prevalent among CKD patients than the general population in different parts of the world. ${ }^{4,5}$ This problem is distinct from hearing loss attributable to hereditary nephritis (Alport's syndrome) and drug ototoxicities from aminoglycosides and high-dose furosemide. ${ }^{5}$ It is therefore recommended that routine hearing assessment should be included in the protocol for managing patients with CKD.

\section{Professor Sampson Antwi FWACP, FGCP, Cert of Nephrol (SA) Paed. Associate Professor and Head, Department of Child Health, Kwame Nkrumah University of Sci- ence and Technology, Kumasi \\ E-mail: kantwisampson@gmail.com \\ Conflict of interest: None declared}

\section{REFERENCES}

1. 1. Maher D, Smeeth L, Sekajugo J. Health transition in Africa: practical policy proposals for primary care. Bull World Health Organ2010; 88:943-948. Doi:10.2471/BLT.10.077891

2. Sherwood PR, Given CW, Given BA, von Eye A. Caregiver burden and depressive symptoms: analysis of common outcomes in caregivers of elderly patients. J Aging Health 2005; 17: 125-147.

3. Beanlands $\mathrm{H}$, Horsburgh ME, Fox S, Howe $\underline{\text { A, Locking-Cusolito } \mathrm{H}}$, Pare $\mathrm{K}$ et al. Caregiving by family and friends of adults receiving dialysis. Nephrol Nurs J. 2005 Nov-Dec;32(6):621-31

4. Meena RS, Aseri Y, Singh BK et al. Hearing loss in patients of chronic renal failure: a study of 100 cases. Indian J Otolaryngol Head Neck Surg; 64:356-9; 2012

5. Kovalik C.E. Endocrine and Neurologic manifestations of kidney failure. In: Greenberg A. Ed. Primer on Kidney Diseases. Pennsylvania: Saunders, $4^{\text {th }}$ edition, 2005:pp527-536 